\title{
Invited Discussion on: Transaxillary Endoscopic approach to Capsular Contracture following Previous Breast Augmentation-Operative Technique and Clinical Outcome
}

\author{
Mitchell Brown ${ }^{1}$ (D)
}

Received: 17 November 2019/Accepted: 20 November 2019/Published online: 2 December 2019

(C) Springer Science+Business Media, LLC, part of Springer Nature and International Society of Aesthetic Plastic Surgery 2019

Level of Evidence $V$ This journal requires that authors assign a level of evidence to each article. For a full description of these Evidence-Based Medicine ratings, please refer to the Table of Contents or the online Instructions to Authors www.springer.com/00266.

This is a very intriguing article regarding the use of a transaxillary (TA) approach under endoscopic control for the management of established Baker grade III and IV capsular contractures. It is timely in the sense that the number of women undergoing secondary or revision breast implant surgery is increasing. At the same time, articles on novel approaches to breast implant revision are under represented in the literature. This article is a welcome addition.

The authors performed a prospective review of women presenting with Baker grade III or IV capsular contracture following previous breast augmentation through a TA approach. The article is coming from an institution in Asia, and it is assumed (although not stated) that all of the patients are of Asian descent. The authors discuss that the choice of a TA approach for breast augmentation is particularly relevant in Asian patients in order to avoid a scar on the actual breast. This same expectation exists when secondary surgery is indicated.

There were a total of 21 patients identified between 2012 and 2017. Bilateral surgery was performed in all patients, resulting in a total of 42 breasts. The indication for surgery was grade III or IV contracture. The authors report

Mitchell Brown

drbrown@torontoplasticsurgery.com

1 Department of Surgery, University of Toronto, 790 Bay Street, Suite 410, Toronto, Ontario M5G 1N8, Canada that 33 breasts had a grade III contracture and 9 breasts had a grade IV contracture. It seems unusual that all 21 patients had contractures in both breasts. Many patients develop contracture on one side only, and so the reader is left to wonder if bilateral contracture was in fact an indication for inclusion or if the authors simply did not see any women in that time period with unilateral contracture.

All patients were treated with endoscopic-assisted total capsulectomy, explantation and implant replacement. The technique is very well described, and the figures augment the description nicely. A video of the technique would be of great value. The authors discuss the use of tumescent infiltration to facilitate dissection. This is not very well described, and it is unclear as to how much fluid is injected and whether the tumescence is placed all around the capsule, on the anterior surface, posterior surface or both. The location that would benefit the most from hydrodissection is on the posterior wall, but it has been my experience that this is a very difficult plane to enter with a needle or a cannula unless the contracture is more severe.

I am in full agreement with the authors that the management of contracture should include total capsulectomy, when possible, and implant replacement. This follows the principle of minimizing the bacterial or biofilm load in the new pocket. I was impressed with the clarity and visualization demonstrated in the figures, and it is evident that the magnification from the use of an endoscope can assist with the precision of this dissection. It is my experience that most advanced capsules dissect fairly well off of the chest wall and that less contracted and thin capsules can be almost impossible to remove entirely without sacrificing rib periosteum and intercostal fascia. The discussion by the authors of using a $2 \times 2$ meshing approach for the removal 
of the posterior wall was valuable and is an excellent suggestion for the capsule that is more adherent.

The authors comment that all patients had their implant pockets converted to a dual plane. Although not clearly stated, the reader must assume that implants were initially placed in a submuscular position with minimal or no release of the inferior border of the pectoralis major muscle. The conversion to dual plane, which is well shown in Figures 7 and 9, involved release of the lower muscle origin and dissection of the gland off of the muscle to facilitate lower pole expansion. The patient shown in Figure 10 demonstrates a nice redistribution of volume with more of the implant sitting between the nipple and the inframammary fold. The authors do not go in to detail on the management of the inframammary fold, but it would be interesting to know if they have had to repair or support the fold using internal sutures or staples. If so, a description of how to perform this through a TA approach would be helpful for the reader.

The follow-up for the 21 patients averaged 13 months with a range of 6 months to two years. This is a reasonable length of time for looking at early recurrence of capsular contracture. It would be nice to know how many of the 21 patients had follow-up of a minimum of one year. The authors state that there were no major complications within the study group. Unfortunately, the authors do not comment specifically on implant malposition, breast asymmetry and inframammary fold malposition. When performing a total capsulectomy and pocket conversion all through a distant access site, the risk of these complications is real. The article would have benefitted from a more in-depth discussion of risks and complications, and specifically steps that the authors took to avoid these from occurring.

The benefits of performing surgery through an incision in the axilla are obvious. Most notably, the surgeon is able to avoid a scar on the breast. Incisions in the inframammary fold or around the areola offer the advantage of having a more direct access to the breast implant pocket.
This is important especially in instances of bleeding or when encountering a ruptured silicone gel-filled breast implant. Although I have not personally attempted secondary implant surgery through the axilla, I would assume that if the surgeon encountered bleeding during capsulectomy, obtaining control of the bleeding may be a challenge. This caveat may be wise for the reader to consider, especially in cases of severe contracture. Patients should likely be consented for additional incisions in the event that uncontrolled bleeding occurs.

Traditionally, a TA approach for breast implant surgery has been reserved for select cases of primary breast augmentation and for simple secondary procedures such as capsulotomy or implant exchange. When using a TA approach, endoscopic assist allows for surgery to be performed under direct vision with principles in keeping with atraumatic dissection, prospective hemostasis and gentle handling of the breast implant. The authors should be congratulated for their excellent demonstration of a TA approach for more complex secondary surgery, namely capsulectomy and implant replacement. The technique is well presented, and their results are impressive.

\section{Compliance with Ethical Standards}

Conflict of interest The author declares that he has no conflict of interest to disclose.

Disclosure The author has no financial disclosures relevant to the content of this article or commentary.

Human and Animal Rights This article does not contain any studies with human participants or animals performed by any of the authors.

Informed Consent For this type of study, informed consent is not required.

Publisher's Note Springer Nature remains neutral with regard to jurisdictional claims in published maps and institutional affiliations. 